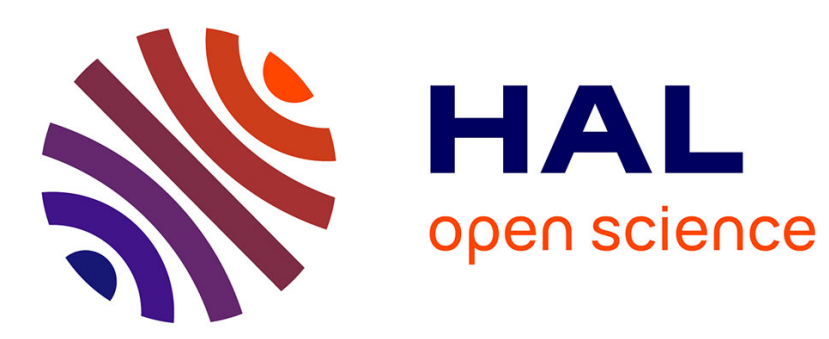

\title{
nD Quasi-Affine Transformations
}

Marie-Andrée Jacob-da Col, Loïc Mazo

\section{To cite this version:}

Marie-Andrée Jacob-da Col, Loïc Mazo. nD Quasi-Affine Transformations. Discrete Geometry for Computer Imagery, Apr 2016, Nantes, France. pp.337-348, 10.1007/978-3-319-32360-2_26 . hal01078723v2

\section{HAL Id: hal-01078723 \\ https://hal.science/hal-01078723v2}

Submitted on 19 Oct 2018

HAL is a multi-disciplinary open access archive for the deposit and dissemination of scientific research documents, whether they are published or not. The documents may come from teaching and research institutions in France or abroad, or from public or private research centers.
L'archive ouverte pluridisciplinaire HAL, est destinée au dépôt et à la diffusion de documents scientifiques de niveau recherche, publiés ou non, émanant des établissements d'enseignement et de recherche français ou étrangers, des laboratoires publics ou privés. 


\title{
nD Quasi-Affine Transformations
}

\author{
Marie-Andrée Jacob-Da Col and Loïc Mazo \\ ICube-UMR 7357, 300 Bd Sébastien Brant - CS10413 \\ 67412 Illkirch Cedex France \\ $<$ dacolm, mazo>@unistra.fr
}

\begin{abstract}
A Quasi-Affine Transformation (QAT) is a transformation on $\mathbb{Z}^{n}$ which corresponds to the composition of a rational affine transformation and an integer part function. The aim of this paper is twofold. Firstly, it brings new insight into the periodic structures involved by a QAT. Secondly, some new results in $n \mathrm{D}$ are presented specifically about the behavior under iteration of a QAT.
\end{abstract}

\section{Introduction.}

Affine transformations (scaling, rotating, etc.) are widely used in image analysis and processing, for instance to register images. Nevertheless, two issues have to be considered when one uses such a transformation in the digital world. Indeed digital images only embed a finite number of pixels (or voxels) and it is well known that for instance continuous rotations when applied on $\mathbb{Z}^{n}$ are generally not bijective [11, 12], nor topology preserving [10] (e.g. holes can appear in a simply connected object). Moreover, very few affine transformations can be exactly calculated in computers. For instance, an 1/8th of a turn involves an irrational coefficient that cannot be represented by a floating number. In order to study the properties of the "affine" transformations embedded in computers, the first author of the present paper has proposed in [4] to model them by Quasi-Affine Transformations (QAT) which are actual affine transformations on $\mathbb{Q}^{n}$ followed by a floor function. We stress that this model slightly differs from the model used for instance by Nouvel et al. or Ngo et al. who consider Euclidean affine transformations (in $\mathbb{R}^{n}$ ) followed by some rounding function.

At this point, we need to introduce a few notations. Points of $\mathbb{R}^{n}$, mixed up with vectors, are denoted with a bold font and, for any $c \in \mathbb{R}, \boldsymbol{c}$ denotes the vector whose coordinates are all equal to $c$ (in particular, $\mathbf{0}$ is the null vector). Binary relations between vectors (e.g. $\boldsymbol{v} \leq \boldsymbol{w}$, or $\boldsymbol{v} \leq \mathbf{0}$ ), are to be understood coordinatewise. The floor function is denoted by $\lfloor\cdot\rfloor$ and, $\omega$ being a positive integer, we write $\lfloor x\rfloor_{\omega}$ for $\left\lfloor\frac{1}{\omega} x\right\rfloor$. If $g: \mathbb{Z}^{n} \rightarrow \mathbb{Z}^{n}$ is an affine transformation of the $n$-dimensional $\mathbb{Z}$-module, we note $g_{0}$ the linear part of $g$ and $v$ its constant part: $g=g_{0}+v$. The Quasi-Affine Transformation (QAT) associated with $g$ and $\omega$ is the transformation $\lfloor g\rfloor_{\omega}: \mathbb{Z}^{n} \rightarrow \mathbb{Z}^{n}$ defined by $\lfloor g\rfloor_{\omega}(\boldsymbol{x})=\left\lfloor\frac{1}{\omega} g(\boldsymbol{x})\right\rfloor$.

We can now explain how a QAT $\lfloor g\rfloor_{\omega}$ can be derived from an Euclidean affine transformation $t$. To fix ideas, let $t$ be the rotation of $\pi / 4$ radians such that $t(\mathbf{0})=(1 / 3,1 / 7)$ and $\boldsymbol{u}$ be the Euclidean vector with coordinates $(\pi, 7 / 3)$. 
The first way to obtain a QAT from the Euclidean rotation $t$ is to approximate the real coefficients of (the matrix of) $t$ by rationals whose greatest common denominator is $\omega$ leading to a rational affine transformation $t^{\prime}: \mathbb{Q}^{n} \rightarrow \mathbb{Q}^{n}$. Then the application of $t^{\prime}$ on the integer vectors $\lfloor\boldsymbol{x}\rfloor, \boldsymbol{x} \in \mathbb{R}^{n}$, followed by the floor function leads to the QAT $\lfloor g\rfloor_{\omega}: \mathbb{Z}^{n} \rightarrow \mathbb{Z}^{n}$ where $g=\omega t^{\prime}$ is an affine transformation on $\mathbb{Z}^{n}$. For instance, one can set $\omega=1000$ and $g(\boldsymbol{x})=g_{0}(\boldsymbol{x})+\boldsymbol{v}$ where $g_{0}$ is defined by its matrix $\left(\begin{array}{cc}707 & -708 \\ 707 & 707\end{array}\right)$ and $\boldsymbol{v}$ is the vector $\left(\begin{array}{l}333 \\ 142\end{array}\right)$. Then, $t(\boldsymbol{u})$ is computed as

$$
\widehat{t(\boldsymbol{u})}=\lfloor g\rfloor_{\omega}(\lfloor\boldsymbol{u}\rfloor)=\left\lfloor\frac{1}{1000}\left(\left(\begin{array}{cc}
707 & -708 \\
707 & 707
\end{array}\right)\left(\begin{array}{l}
3 \\
2
\end{array}\right)+\left(\begin{array}{l}
333 \\
142
\end{array}\right)\right)\right\rfloor .
$$

We have proposed in [1] another way to discretize an affine transformation in order to introduce the multigrid convergence scheme in the framework of QATs. Firstly, each real number is 'projected' on the integer line by a scaling operation followed by a rounding as in $\pi \rightarrow\lfloor\omega \pi\rfloor=3141$ taking $\omega=1000$ as the scaling factor $^{1}$. Then, any multiplication must be followed by an integer division. Indeed, consider for instance the calculus $\sqrt{2} \times \pi+6 / 7$ with the precision $\omega=1000$. This calculation can be done by $\lfloor 1414 \times 3141\rfloor_{1000}+857=4441+857=5298$. Then, a "back-projection" on the real line gives the final result 5.298. In this setting, the Euclidean transformation $t$ corresponds to the integer affine transformation $\lfloor g\rfloor_{\omega}: \boldsymbol{x} \mapsto\left\lfloor g_{0}(\boldsymbol{x})\right\rfloor_{\omega}+\boldsymbol{v}$, that is to the QAT $\left\lfloor g_{0}+\omega \boldsymbol{v}\right\rfloor_{\omega}$ and $t(\boldsymbol{u})$ is computed as

$$
\widehat{t(\boldsymbol{u})}=\frac{1}{\omega}\left(\lfloor g\rfloor_{\omega}(\lfloor\omega \boldsymbol{u}\rfloor)\right)=\frac{1}{1000}\left\lfloor\frac{1}{1000}\left(\begin{array}{cc}
707 & -708 \\
707 & 707
\end{array}\right)\left(\begin{array}{l}
3141 \\
2333
\end{array}\right)+\left(\begin{array}{l}
333 \\
142
\end{array}\right)\right\rfloor .
$$

However, here it doesn't matter what the discretization scheme is. Indeed, the present paper is only interested in the properties of the QATs by themselves. More precisely, it is devoted to the understanding of the periodic structure created by a QAT, which basically expresses the lack of bijectivity of the transformation, and on the behavior under iteration, which brings out the loss of precision. We present new results about these two topics in the general case and also give a new perspective on the links with the lattice group theory. The article is organized as follows. In Sect. 2, we study tilings generated by QATs: a tile is the set of the inverse images of a given point. Indeed, as explained above, a QAT derived from a bijective affine transformation in the Euclidean world will generally not be bijective and fibers can be empty, contain one or several points. Then, if we only focus on the difference between the affine transformation and the quasi-affine transformation, tiles yield a lattice structure in the discrete space. In 2D and 3D, explicit formulas have been proposed to compute a minimal basis of the QAT periodic structure $[6,3]$. In the $n$-dimensional case, an upper bound on the number of distinct tiles is given in [5]. We now give a closed formula for the period in any dimension. In Sect. 3, we study the behavior under iterations of a contracting QAT. If $g$ is a contracting affine transformation of $\mathbb{R}^{n}$ then $g$ has a unique fixed point and for each $\boldsymbol{x} \in \mathbb{R}^{n}$ the sequence $g^{n}(X)$ tends toward this fixed point. But the corresponding QAT has not necessarily a unique

\footnotetext{
${ }^{1}$ One can use two distinct scale factors for the digitization of the space and the quantification of the transformation.
} 


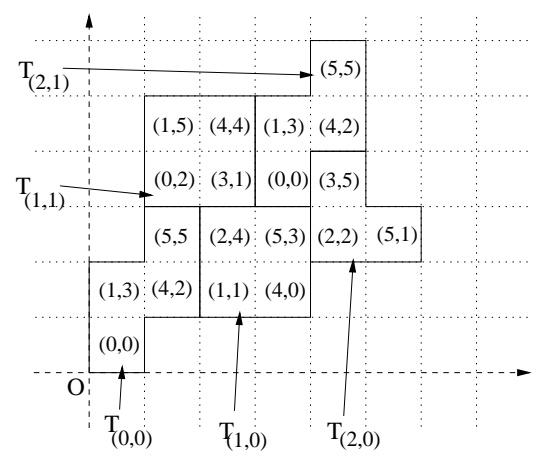

Fig. 1: Example of tiles and remainders. A point of $\mathbb{Z}^{2}$ is represented by a unit square whose bottom-left corner corresponds to the represented point. For each point in a tile we provide its corresponding remainder. Tiles $T_{(2,1)}$ and $T_{(0,0)}$ are arithmetically equivalent. The tiles $T_{(1,0)}$ and $T_{(1,1)}$ have the same shapes but they are not equivalent.

fixed point. The behavior under iteration of the 2D contracting QATs has been studied in [4], [8] and [9]. In this paper, we study the $n \mathrm{D}$ case and prove that a QAT has a unique fixed point if and only if there are no cycle among the points of norm 1 .

\section{Periodicity of the tiles}

In order to obtain efficient computations for the affine transformation of discrete images, we can use some properties of QATs. In [7] the 2D case has been treated and extended to 3D in [3]. More precisely, we have seen in these papers that the periodicity properties of the tilings associated to QATs improve considerably the computation of the discrete affine transformations. This periodicity has been studied in the n-dimensional case in [5] and [2]. Nevertheless, no closed formula for the tile period was given in these papers. We begin by recalling some definitions about QATs and their tiles.

A nonsingular QAT, that is a QAT derived from a bijective affine transformation, is generally not bijective and for a given QAT $\lfloor g\rfloor_{\omega}$, the preimages $\lfloor g\rfloor_{\omega}^{-1}(\boldsymbol{y})$ can have either none, one or several elements. As these preimages define a tessellation of the space $\mathbb{Z}^{n}$, we call them tiles. In the sequel we assume a QAT $\lfloor g\rfloor_{\omega}=\left\lfloor\frac{1}{\omega}\left(g_{0}+v\right)\right\rfloor$ and we note $T_{\boldsymbol{y}}$ the tile of $\boldsymbol{y}$ for this QAT. The remainder of a tile $T_{\boldsymbol{y}}$, noted $r_{\boldsymbol{y}}$, is the set of the remainders modulo $\omega$ of the vectors $g(\boldsymbol{x}), \boldsymbol{x} \in T_{\boldsymbol{y}}$, that is

$$
r_{\boldsymbol{y}}=\left\{g(\boldsymbol{x})-\omega \boldsymbol{y} \mid x \in T_{\boldsymbol{y}}\right\} .
$$

Observe that the sets $T_{\boldsymbol{y}}$ and $r_{\boldsymbol{y}}$ have the same cardinal since $g$ is nonsingular. Two tiles $T_{\boldsymbol{y}}$ and $T_{\boldsymbol{y}^{\prime}}$ are said to be (arithmetically) equivalent when their remainders are the same. We then write $T_{\boldsymbol{y}} \equiv T_{\boldsymbol{y}^{\prime}}$. Two vectors $\boldsymbol{y}$ and $\boldsymbol{y}^{\prime}$ of $\mathbb{Z}^{n}$ are 
equivalent modulo $\lfloor g\rfloor_{\omega}$ - we write $\boldsymbol{y} \equiv \boldsymbol{y}^{\prime}$ - if the tiles $T_{\boldsymbol{y}}$ and $T_{\boldsymbol{y}^{\prime}}$ are equivalent. Eventually, we write $p \wedge q$, resp. $p \vee q$, for the gcd, resp. the lcm, of the integers $p$ and $q$. Two equivalent tiles of a QAT $\lfloor g\rfloor_{\omega}$ share the same shape but the converse is false. Figure 1 exhibits some tiles of the QAT defined by the integer matrix $\left(\begin{array}{cc}3 & 1 \\ -1 & 3\end{array}\right)$, the vector $\boldsymbol{v}=\mathbf{0}$ and the integer $\omega=6$.

The next proposition and its corollary describes the periodic structures induced by the equivalence modulo $\lfloor g\rfloor_{\omega}$ and the corresponding tile equivalence.

Proposition 1. Let $\lfloor g\rfloor_{\omega}$ be a non singular QAT. The equivalence modulo $\lfloor g\rfloor_{\omega}$ has a translational symmetry whose lattice group is $G=g_{0}\left(\frac{1}{\omega} \mathbb{Z}^{n}\right) \cap \mathbb{Z}^{n}$. In particular, two non empty tiles $T_{\boldsymbol{y}}$ and $T_{\boldsymbol{y}^{\prime}}$ are equivalent iff $\boldsymbol{y}-\boldsymbol{y}^{\prime} \in \frac{1}{\omega} g_{0}\left(\mathbb{Z}^{n}\right)$. Moreover, the multi-valued function defined by $\boldsymbol{q} \mapsto T_{\lfloor\boldsymbol{q}\rfloor}$ induces a surjection from the quotient space $\left(\frac{1}{\omega} g\left(\mathbb{Z}^{n}\right) \cup \mathbb{Z}^{n}\right) / G$ onto the tile classe set $\left\{T_{\boldsymbol{y}}\right\}_{\boldsymbol{y} \in \mathbb{Z}^{n}} / \equiv$.

Proof. Let $\boldsymbol{y}, \boldsymbol{y}^{\prime}$ in $\mathbb{Z}^{n}$ such that $\boldsymbol{y}^{\prime}-\boldsymbol{y}=\frac{1}{\omega} g_{0}(\boldsymbol{z})$ for some $\boldsymbol{z} \in \mathbb{Z}^{n}$. We shall prove that the tiles $T_{\boldsymbol{y}}$ and $T_{\boldsymbol{y}^{\prime}}$ have the same remainders. Let $\boldsymbol{x} \in T_{\boldsymbol{y}}$, if such an integer vector exists. The remainder of $g(\boldsymbol{x})$ modulo $\omega$ is $r(\boldsymbol{x})=g(\boldsymbol{x})-\boldsymbol{\omega} \boldsymbol{y}$. We observe that $\frac{1}{\omega} g(\boldsymbol{x}+\boldsymbol{z})-\boldsymbol{y}^{\prime}=\frac{1}{\omega} g(\boldsymbol{x})+\frac{1}{\omega} g_{0}(\boldsymbol{z})-\boldsymbol{y}^{\prime}=\frac{1}{\omega} g(\boldsymbol{x})-\boldsymbol{y}=\frac{1}{\omega} r(\boldsymbol{x})$ by definition of $\boldsymbol{z}$ and $r(\boldsymbol{x})$. Thus, the integer vector $\boldsymbol{x}^{\prime}=\boldsymbol{x}+\boldsymbol{z}$ lies in $T_{\boldsymbol{y}^{\prime}}$ and the remainder of $g\left(\boldsymbol{x}^{\prime}\right)$ modulo $\omega$ is equal to the remainder of $g(\boldsymbol{x})$ modulo $\omega$. Thereby, we proved that either $r_{\boldsymbol{y}}=\emptyset$ or $\emptyset \neq r_{\boldsymbol{y}} \subseteq r_{\boldsymbol{y}^{\prime}}$. It is plain that, by the same reasoning, we can also prove $r_{\boldsymbol{y}^{\prime}}=\emptyset$ or $\emptyset \neq r_{\boldsymbol{y}^{\prime}} \subseteq r_{\boldsymbol{y}}$. Hence, either $T_{\boldsymbol{y}}=T_{\boldsymbol{y}^{\prime}}=\emptyset$ or $r_{\boldsymbol{y}}=r_{\boldsymbol{y}^{\prime}}$, that is, in both cases, $T_{\boldsymbol{y}} \equiv T_{\boldsymbol{y}^{\prime}}$.

Conversely, if two non empty tiles $T_{\boldsymbol{y}}$ and $T_{\boldsymbol{y}^{\prime}}$ are equivalent, there exist two vectors $\boldsymbol{x} \in T_{\boldsymbol{y}}$ and $\boldsymbol{x}^{\prime} \in T_{\boldsymbol{y}^{\prime}}$ such that $g(\boldsymbol{x}) \equiv g\left(\boldsymbol{x}^{\prime}\right)(\bmod \omega)$. Thus $g_{0}\left(\boldsymbol{x}^{\prime}-\boldsymbol{x}\right) \equiv$ $\mathbf{0}(\bmod \omega)$ so $\boldsymbol{z}=\frac{1}{\omega} g_{0}\left(\boldsymbol{x}^{\prime}-\boldsymbol{x}\right) \in \mathbb{Z}^{n}$. Then, $\left\lfloor g\left(\boldsymbol{x}^{\prime}\right)\right\rfloor \omega=\left\lfloor\frac{1}{\omega}\left(g(\boldsymbol{x})+g_{0}\left(\boldsymbol{x}^{\prime}-\boldsymbol{x}\right)\right)\right\rfloor=$ $\left\lfloor\frac{1}{\omega} g(\boldsymbol{x})+\boldsymbol{z}\right\rfloor=\left\lfloor\frac{1}{\omega} g(\boldsymbol{x})\right\rfloor+\boldsymbol{z}=\boldsymbol{y}+\boldsymbol{z}$ where $\boldsymbol{z} \in \frac{1}{\omega} g_{0}\left(\mathbb{Z}^{n}\right)$. We cannot assert that for two empty tiles $T_{\boldsymbol{y}}$ and $T_{\boldsymbol{y}^{\prime}}$ there exists $\boldsymbol{z} \in \frac{1}{\omega} g_{0}\left(\mathbb{Z}^{n}\right)$ such that $\boldsymbol{y}^{\prime}=\boldsymbol{y}+\boldsymbol{z}$. Nevertheless, this is not necessary to obtain the result since the tiles cannot all be empty.

Incidentally, we have shown that, for any $\boldsymbol{x}, \boldsymbol{x}^{\prime}$ in $\mathbb{Z}^{n}$, if $g(\boldsymbol{x}) \equiv g\left(\boldsymbol{x}^{\prime}\right)(\bmod \omega)$, then $\left\lfloor g\left(\boldsymbol{x}^{\prime}\right)\right\rfloor_{\omega}=\lfloor g(\boldsymbol{x})\rfloor_{\omega}+\boldsymbol{z}$ where $\boldsymbol{z} \in \frac{1}{\omega} g_{0}\left(\mathbb{Z}^{n}\right)$ which, from the first part of the proof, implies that $T_{\left\lfloor g\left(\boldsymbol{x}^{\prime}\right)\right\rfloor_{\omega}} \equiv T_{\lfloor g(\boldsymbol{x})\rfloor_{\omega}}$.

Note that, from a practical point of view, the last assertion of Prop. 1 means that one can prove that two tiles are equivalent by just exhibiting two integer points in these tiles whose images under $\frac{1}{\omega} g$ have the same fractional part.

Corollary 1. Let $\lfloor g\rfloor_{\omega}$ be a non singular QAT. The tiles of $\lfloor g\rfloor_{\omega}$ have a translational symmetry whose lattice group is $g_{0}^{-1}\left(\omega \mathbb{Z}^{n}\right) \cap \mathbb{Z}^{n}$.

Proof. Let $\boldsymbol{y}, \boldsymbol{z} \in \mathbb{Z}^{n}$ and $t$ the translation of vector $g_{0}^{-1}(\omega \boldsymbol{z})$. Assume that the tiles $T_{\boldsymbol{y}}$ and $T_{\boldsymbol{y}+\boldsymbol{z}}$ are equivalent. Then $r_{\boldsymbol{y}}=r_{\boldsymbol{y}+\boldsymbol{z}}$ and

$$
\begin{aligned}
T_{\boldsymbol{y}+\boldsymbol{z}} & =\left\{g^{-1}(\omega(\boldsymbol{y}+\boldsymbol{z})+\boldsymbol{r}) \mid \boldsymbol{r} \in r_{\boldsymbol{y}+\boldsymbol{z}}\right\} \\
& =\left\{g^{-1}(\omega(\boldsymbol{y}+\boldsymbol{z})+\boldsymbol{r}) \mid \boldsymbol{r} \in r_{\boldsymbol{y}}\right\} \\
& =\left\{g^{-1}(\omega \boldsymbol{y}+\boldsymbol{r})+g_{0}^{-1}(\omega \boldsymbol{z}) \mid \boldsymbol{r} \in r_{\boldsymbol{y}}\right\} \\
& =t\left(T_{\boldsymbol{y}}\right) .
\end{aligned}
$$


In particular, $g_{0}^{-1}(\omega \boldsymbol{z})$ is an integer vector.

Conversely, let $\boldsymbol{y}, \boldsymbol{u} \in \mathbb{Z}^{n}$ and let $t$ be the translation of vector $\boldsymbol{u}$. Assume that there exists $\boldsymbol{z} \in \mathbb{Z}^{n}$ such that $\boldsymbol{u}=g_{0}^{-1}(\boldsymbol{\omega} \boldsymbol{z})$. Since $\boldsymbol{z}=\frac{1}{\omega} g_{0}(\boldsymbol{u})$, we derive from Prop. 1 that $T_{\boldsymbol{y}+\boldsymbol{z}} \equiv T_{\boldsymbol{y}}$. Then, from the first part of the proof, $t\left(T_{\boldsymbol{y}}\right)=T_{\boldsymbol{y}+\boldsymbol{z}}$. Thus, $t\left(T_{\boldsymbol{y}}\right)$ is a tile and this tile is equivalent to $T_{\boldsymbol{y}}$.

In [5], the authors give upper and lower bounds for the number of tile equivalent classes. Thanks to the Smith normal form of integer matrices, we give hereafter the exact number of classes. We also provide the cardinal of any (maximal) union of representative tiles. Firstly, we give two lemmas that describe the role of unimodular transformations on the lattices associated to a QAT.

Lemma 1 ([5]). Let $u$ be an unimodular transformation. The $Q A T\lfloor g\rfloor_{\omega}$ and $\lfloor g \circ u\rfloor_{\omega}$ have the same tile remainders. In particular, they share the same tile equivalence and the same empty tiles.

Proof. We note $T_{\boldsymbol{y}}^{u}$, resp. $r_{\boldsymbol{y}}^{u}$, the tile of $\boldsymbol{y}$, resp. the remainder of $T_{\boldsymbol{y}}^{u}$, for the QAT $\lfloor g \circ u\rfloor_{\omega}$ while $T_{\boldsymbol{y}}$ and $r_{\boldsymbol{y}}$ are the tile of $\boldsymbol{y}$, resp. the remainder of $T_{\boldsymbol{y}}$, for the QAT $\lfloor g\rfloor_{\omega}$. We have $\boldsymbol{x} \in T_{\boldsymbol{y}}^{u} \Longleftrightarrow u(\boldsymbol{x}) \in T_{\boldsymbol{y}}$, thus $T_{\boldsymbol{y}}=u\left(T_{\boldsymbol{y}}^{u}\right)$. Furthermore,

$$
r_{\boldsymbol{y}}^{u}=\left\{g \circ u(\boldsymbol{x})-\omega \boldsymbol{y} \mid \boldsymbol{x} \in T_{\boldsymbol{y}}^{u}\right\}=\left\{g(\boldsymbol{z})-\omega \boldsymbol{y} \mid \boldsymbol{z} \in T_{\boldsymbol{y}}\right\}=r_{\boldsymbol{y}} .
$$

Lemma 2. Let $u$ be an unimodular transformation. The cardinals of the fundamental domains of the equivalences modulo $\lfloor g\rfloor_{\omega}$ and modulo $\lfloor u \circ g\rfloor_{\omega}$ are equal.

Proof. The lattice groups of the equivalences modulo $\lfloor g\rfloor_{\omega}$ and modulo $\lfloor u \circ g\rfloor_{\omega}$ are $L_{1}=\mathbb{Z}^{n} \cap \frac{1}{\omega} g_{0}\left(\mathbb{Z}^{n}\right)$ and $L_{2}=\mathbb{Z}^{n} \cap \frac{1}{\omega} u \circ g_{0}\left(\mathbb{Z}^{n}\right)$. Note that $L_{2}=u\left(L_{1}\right)$ since $u\left(\mathbb{Z}^{n}\right)=\mathbb{Z}^{n}$ and $u$ is bijective $(u$ is unimodular). As $|\operatorname{det}(u)|=1$, we get that the volumes of the parallelepipeds wrapping $L_{1}$ and $L_{2}$ are equal. Then, from Picks's theorem, we derive that the numbers of integer vectors in these domains are identical.

The following lemma describes the lattice groups of QATs whose matrix is diagonal.

Lemma 3. Let $\lfloor g\rfloor_{\omega}$ be a nonsingular QAT whose matrix $\left(d_{i, j}\right)$ is diagonal. Then,

- the lattice group of the equivalence modulo $\lfloor g\rfloor_{\omega}$ is $\prod_{i=1}^{n}\left(\frac{d_{i, i}}{\omega \wedge d_{i, i}}\right) \mathbb{Z}$;

- the lattice group of the non empty tiles is $\prod_{i=1}^{n}\left(\frac{\omega}{\omega \wedge d_{i, i}}\right) \mathbb{Z}$.

Proof.

- From Prop. 1, the lattice group of the equivalence modulo $\lfloor g\rfloor_{\omega}$ is $\mathbb{Z}^{n} \cap$ $g_{0}\left(\frac{1}{\omega} \mathbb{Z}^{n}\right)=\frac{1}{\omega}\left(\omega \mathbb{Z}^{n} \cap g_{0}\left(\mathbb{Z}^{n}\right)\right)$ and $g_{0}\left(\mathbb{Z}^{n}\right)=\prod_{i=1}^{n}\left(d_{i, i} \mathbb{Z}\right)$ for $g_{0}$ is diagonal. Then, the relation $\omega \vee d_{i, i}=\left(\omega d_{i, i}\right) /\left(\omega \wedge d_{i, i}\right)$ yields the first part of the result.

- From Corollary. 1, the lattice group of the non empty tiles is $g_{0}^{-1}\left(\omega \mathbb{Z}^{n}\right) \cap \mathbb{Z}^{n}=$ $\prod_{i=1}^{n} \frac{1}{d_{i, i}}\left(\omega \vee d_{i, i}\right) \mathbb{Z}=\prod_{i=1}^{n} \frac{\omega}{\omega \wedge d_{i, i}} \mathbb{Z}$. 
Thanks to the Smith normal form of the affine transformations of $\mathbb{Z}^{n}$, we now derive from the three preceding lemmas the cardinal of the fundamental domains of the lattice structures involved in a QAT.

Theorem 1. Let $\lfloor g\rfloor_{\omega}$ be a nonsingular $Q A T$ and $D$ be a fundamental domain of the equivalence modulo $\lfloor g\rfloor_{\omega}$. Noting $s=\left(s_{i, j}\right)$ the Smith normal form of the matrix of $g_{0}$ and $\delta$ its determinant, one has

$$
\# D=\frac{\delta}{\prod_{i=1}^{n} \omega \wedge s_{i, i}} \quad \text { and } \quad \sum_{y \in D} \# T_{y}=\frac{\omega^{n}}{\prod_{i=1}^{n} \omega \wedge s_{i, i}} .
$$

Proof.

- From Prop. 1, the cardinal of $D$ only depends on $g_{0}$. Let $s=u \circ g_{0} \circ v$ be the Smith normal form of $g_{0}$. From Lemmas 1 and 2, we derive that $\# D=\# D^{\prime}$ where $D^{\prime}$ is the fundamental domain of the equivalence modulo $\lfloor s\rfloor_{\omega}$. Then Lemma 3 gives the first result.

- From Corollary 1, $\sum_{y \in D} \# T_{y}$ is equal to the cardinal of the fundamental domain of $g_{0}^{-1}\left(\omega \mathbb{Z}^{n}\right) \cap \mathbb{Z}^{n}$. Then, thanks to the properties of unimodular transformations, we have

$$
\begin{aligned}
g_{0}^{-1}\left(\omega \mathbb{Z}^{n}\right) \cap \mathbb{Z}^{n} & =\left(v \circ s^{-1} \circ u\right)\left(\omega \mathbb{Z}^{n}\right) \cap \mathbb{Z}^{n} \\
& =\left(v \circ s^{-1}\right)\left(\omega \mathbb{Z}^{n}\right) \cap \mathbb{Z}^{n} \\
& =v\left(s^{-1}\left(\omega \mathbb{Z}^{n}\right) \cap v^{-1}\left(\mathbb{Z}^{n}\right)\right) \\
& =v\left(s^{-1}\left(\omega \mathbb{Z}^{n}\right) \cap \mathbb{Z}^{n}\right) .
\end{aligned}
$$

Hence, the lattice of the non empty tiles of $g$ is the image by an unimodular transformation of the non empty tile lattice of $s$. We conclude straightforwardly by invoking the second part of Lemma 3 .

Example : Figure 2 illustrates a QAT in $\mathbb{Z}^{2}\left(g_{0}:\left(\begin{array}{cc}12 & -11 \\ 18 & 36\end{array}\right), \boldsymbol{v}=\mathbf{0}, \boldsymbol{\omega}=84\right)$ whose fundamental domain contains 15 tiles (the tiles with same color are equivalent). In this example, for all $i, j \in \mathbb{N}, T_{(i+5, j)} \equiv T_{(i, j)}$ and $T_{(i+2, j-3)} \equiv T_{(i, j)}$. The fundamental domain of this QAT is the set $\left\{T_{(i, j)} \mid i=0,1,2,3,4, j=0,1,2\right\}$. The Smith normal form of the QAT is $\left(\begin{array}{cc}1 & 0 \\ 0 & 630\end{array}\right)$. From Th. 1, we derive that there is no empty tile $(\# D=15)$ and there are 168 integer points in $\bigcup_{0 \leq i \leq 4,0 \leq j \leq 2} T_{(i, j)}$.

In [3] we used the periodicity to improve the transformation of a $3 \mathrm{D}$ image by a linear transformation. In order to use this periodicity we need to determine a basis of the equivalence modulo $\lfloor g\rfloor_{\omega}$ lattice group such that, for any $m \leq n$, $\left(\boldsymbol{y}_{\boldsymbol{i}}\right)_{i=1}^{m}$ is a basis of $\mathbb{Z}^{m} \times\{\mathbf{0}\}^{n-m}$.

Proposition 2. Let $\lfloor g\rfloor_{\omega}$ be a non singular QAT. There exists a basis $\left(\boldsymbol{y}_{\boldsymbol{i}}\right)_{i=1}^{n}$ of the equivalence modulo $\lfloor g\rfloor_{\omega}$ lattice group such that, for any $m \leq n,\left(\boldsymbol{y}_{i}\right)_{i=1}^{m}$ is 


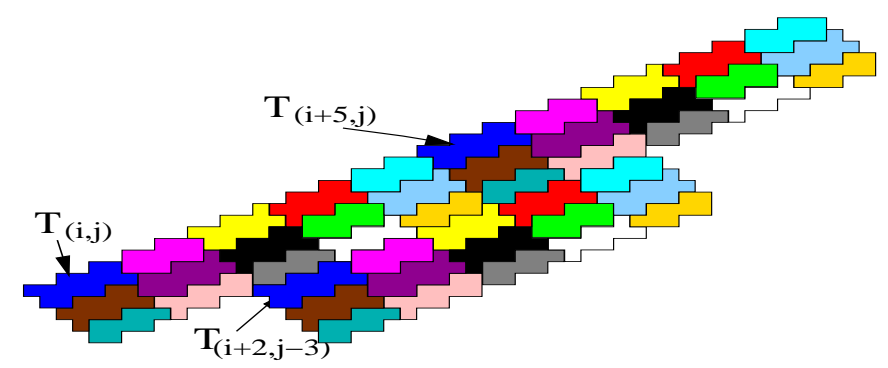

Fig. 2: Periodicity of thes tiles of a 2D QAT (see text)

a basis of $\mathbb{Z}^{m}$ (more specifically, $\mathbb{Z}^{m} \times\{\mathbf{0}\}^{n-m}$ that we identify with $\mathbb{Z}^{m}$ ). Each vector $\boldsymbol{y}_{\boldsymbol{i}}, 1 \leq i \leq n$, is defined by $\boldsymbol{y}_{\boldsymbol{i}}=h_{i}\left(\boldsymbol{\lambda}_{\boldsymbol{i}}\right)$ where $h_{i}$ is the restriction to $\mathbb{Z}^{i}$ of the Hermite normal form of $g_{0}$ and $\boldsymbol{\lambda}_{\boldsymbol{i}}=\left(\lambda_{i, 1}, \ldots, \lambda_{i, i}\right)$ is the solution of $h_{i}\left(\boldsymbol{\lambda}_{\boldsymbol{i}}\right)=\mathbf{0}(\bmod \omega)$ such that $\lambda_{i, i}$ is minimal.

Proof. The modulo $\lfloor g\rfloor_{\omega}$ equivalence lattice group is $g_{0}\left(\frac{1}{\omega} \mathbb{Z}^{n}\right) \cap \mathbb{Z}^{n}$. Let $h$ be the Hermite normal form of $g_{0}$. As $h^{-1} \circ g_{0}$ is unimodular, the group $g_{0}\left(\mathbb{Z}^{n}\right)$ is generated by the column vectors of $h$. Let $\boldsymbol{c}_{\boldsymbol{j}}=\left(h_{i, j}\right)_{i=1}^{n}$ be the $j$-th column vector of $h$. Note that, since $h$ is triangular, $\boldsymbol{c}_{\boldsymbol{j}} \in \mathbb{Z}^{j} \times\{\mathbf{0}\}^{n-j}$. Then finding the basis $\left(\boldsymbol{y}_{\boldsymbol{i}}\right)$ amounts to find the integer tuples $\left(\lambda_{i, 1}, \ldots, \lambda_{i, i}\right)$ such that $\boldsymbol{y}_{\boldsymbol{i}}=$ $\sum_{j=1}^{i} \lambda_{i, j} \boldsymbol{c}_{\boldsymbol{j}} \in \omega \mathbb{Z}^{n}$ and $\lambda_{i, i}$ is minimal.

Proposition 2 shows that we can compute the basis $\left(\boldsymbol{y}_{\boldsymbol{i}}\right)_{i=1}^{n}$ by solving triangular systems of $i$ linear equations in the modules $(\mathbb{Z} / \omega \mathbb{Z})^{i}, 1 \leq i \leq n$, which can be done by Gaussian elimination in polynomial time.

\section{Behavior under iteration of a quasi-linear transformation}

In this section we consider affine transformations $g$ whose vector $\boldsymbol{v}$ is such that $\mathbf{0} \leq \boldsymbol{v}<\boldsymbol{\omega}$. Then, the associated QAT can be seen as the composition of a linear transformation with some rounding operator. For this reason, we say that such a QAT is a quasi linear transformation (QLT). Since we are interested in fixed points, or more generally in cycles, we restrict our study to non expansive transformations for the infinite norm, which is defined on a linear transformation $f$ whose matrix is $A=\left(a_{i, j}\right)$ by $\|f\|_{\infty}=\|A\|_{\infty}=\max _{i}\left(\sum_{j}\left|a_{i, j}\right|\right)$. Thus we have for any vector $\boldsymbol{x},\|f(\boldsymbol{x})\|_{\infty} \leq\|f\|_{\infty} \times\|\boldsymbol{x}\|_{\infty}$. It is well known that if $f$ is a contracting linear transformation of $\mathbb{R}^{n}$ then $f$ has the origin as unique fixed point and for each $\boldsymbol{x} \in \mathbb{R}^{n}$ the sequence $f^{n}(\boldsymbol{x})$ tends toward this fixed point. But a QLT built from a non expansive linear transformation has not necessarily a unique fixed point. Consider the sequence $\boldsymbol{y}_{n}=\left(\lfloor g\rfloor_{\omega}\left(\boldsymbol{y}_{n-1}\right)\right)_{n \geq 0}=$ $\left(\lfloor g\rfloor_{\omega}^{n}\left(\boldsymbol{y}_{0}\right)\right)_{n \geq 0}$ with $\boldsymbol{y}_{0} \in \mathbb{Z}^{n}$. Depending on $\boldsymbol{y}_{0}$ we can obtain a cycle $\left(\boldsymbol{y}_{p}=\boldsymbol{y}_{q}\right.$ for some integer pair $p<q)$, a fixed point $\left(\boldsymbol{y}_{n}=\boldsymbol{y}_{n+1}\right.$ for some $\left.n \in \mathbb{N}\right)$, or leaves 


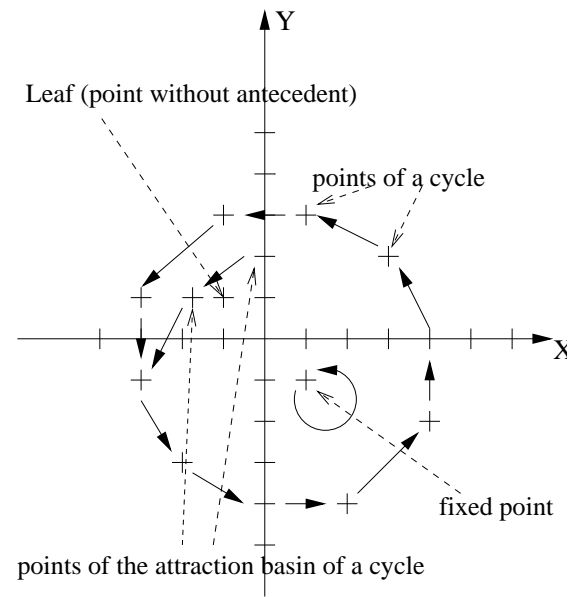

(a)

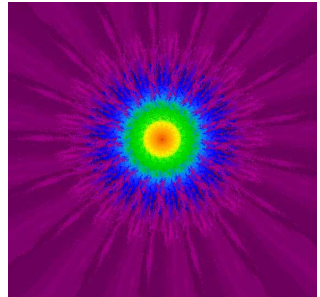

(b)

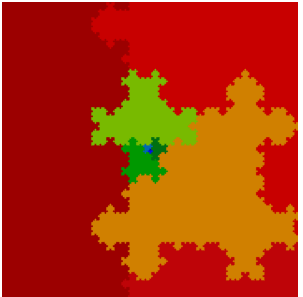

(c)

Fig. 3: (a) Example of cycle, fixed point and leaf. (b) QLT with many cycles. The points of a cycle and those that reach this cycle have the same colours. (c) QLT with a unique fixed point. The colour of a point is determined by the number of iterations necessary to reach the fixed point.

(points without antecedent). Figures $3 \mathrm{a}, 3 \mathrm{~b}$ and $3 \mathrm{c}$ illustrate this behavior. In Fig. 3b the QLT has many cycles. It is defined by $\boldsymbol{y}=\frac{1}{4495}\left(\begin{array}{ccc}4187 & -1622 \\ 1622 & 4187\end{array}\right) \boldsymbol{x}$. In Fig. 3c the QLT which is defined by $\boldsymbol{y}=\frac{1}{3}\left(\begin{array}{cc}-1 & 1 \\ -1 & -1\end{array}\right) \boldsymbol{x}$ has a unique fixed point.

The first results concern the localisation of the cycles. Firstly, the following lemma gives a bound on the difference between a QLT and the corresponding linear transformation.

Lemma 4. Let $\lfloor g\rfloor_{\omega}$ be a $Q L T, d=\lfloor g\rfloor_{\omega}-\frac{1}{\omega} g_{0}$ and $s=\max \left(\frac{\|\boldsymbol{v}\|_{\infty}}{\omega}, 1-\frac{\|\boldsymbol{v}\|_{\infty}}{\omega}\right)$. Then, for any integer vector $\boldsymbol{x},\|d(\boldsymbol{x})\|_{\infty} \leq s$, the inequality being strict if $s>\frac{1}{2}$. 
Proof. Consider $\boldsymbol{x} \in \mathbb{Z}^{n}, \boldsymbol{y}=\lfloor g\rfloor_{\omega}(\boldsymbol{x}) \in \mathbb{Z}^{n}, \boldsymbol{y}^{\prime}=\frac{1}{\omega} g_{0}(\boldsymbol{x}) \in \mathbb{Q}^{n}$ and $d(\boldsymbol{x})=$ $\boldsymbol{y}-\boldsymbol{y}^{\prime}$. We have

$$
\begin{aligned}
d(\boldsymbol{x}) & =\left\lfloor\frac{1}{\omega}\left(g_{0}(\boldsymbol{x})+\boldsymbol{v}\right)\right\rfloor-\frac{1}{\omega} g_{0}(\boldsymbol{x}) \\
& =\frac{1}{\omega}\left(g_{0}(\boldsymbol{x})+\boldsymbol{v}\right)-\boldsymbol{r}-\frac{1}{\omega} g_{0}(\boldsymbol{x}) \text { with } \mathbf{0} \leq \boldsymbol{r}<\mathbf{1} \\
& =\frac{1}{\omega} \boldsymbol{v}-\boldsymbol{r} \text { with } \mathbf{0} \leq \boldsymbol{r}<\mathbf{1}
\end{aligned}
$$

It follows that $\frac{1}{\omega} \boldsymbol{v}-\mathbf{1}<d(\boldsymbol{x}) \leq \frac{1}{\omega} \boldsymbol{v}$. As we assume in the current section that $\mathbf{0} \leq \boldsymbol{v}<\boldsymbol{\omega}$, we get $\|d(\boldsymbol{x})\|_{\infty} \leq s$, the inequality being strict if $s=1-\frac{1}{\omega}\|\boldsymbol{v}\|_{\infty}$ , that is to say if $\|\boldsymbol{v}\|_{\infty}<\frac{\omega}{2}$.

As a consequence of Lemma 4, we show that a QLT corresponding to a non expansive linear transformation is itself non expansive from the origin.

Corollary 2. If $\lfloor g\rfloor_{\omega}$ is a $Q L T$ and $\left\|\frac{1}{\omega} g_{0}\right\|_{\infty} \leq 1$, then $\left\|\lfloor g\rfloor_{\omega}(\boldsymbol{x})\right\|_{\infty} \leq\|\boldsymbol{x}\|_{\infty}$ for any $\boldsymbol{x} \in \mathbb{Z}^{n}$.

Proof. Let $\lfloor g\rfloor_{\omega}$ be a QLT such that $\left\|\frac{1}{\omega} g_{0}\right\|_{\infty} \leq 1$. With the notations of Lemma 4 , we have:

$$
\begin{aligned}
\left\|\lfloor g\rfloor_{\omega}(\boldsymbol{x})\right\|_{\infty} & =\left\|\frac{1}{\omega} g_{0}(\boldsymbol{x})+d(\boldsymbol{x})\right\|_{\infty} \\
& \leq\left\|\frac{1}{\omega} g_{0}(\boldsymbol{x})\right\|_{\infty}+\|d(\boldsymbol{x})\|_{\infty} \\
& \leq \frac{1}{\omega}\left\|g_{0}\right\|_{\infty}\|\boldsymbol{x}\|_{\infty}+\|d(\boldsymbol{x})\|_{\infty} \\
& \leq\|\boldsymbol{x}\|_{\infty}+\|d(\boldsymbol{x})\|_{\infty} \\
& <\|\boldsymbol{x}\|_{\infty}+1 \quad(\text { from Lemma } 4) \\
& \leq\|\boldsymbol{x}\|_{\infty} \text { for }\left\|\lfloor g\rfloor_{\omega}(\boldsymbol{x})\right\|_{\infty} \text { and }\|\boldsymbol{x}\|_{\infty} \text { are integers } .
\end{aligned}
$$

Remark 1. From Corollary 2, we derive that, if $\lfloor g\rfloor_{\omega}$ is a QLT, $\frac{1}{\omega} g_{0}$ is non expansive and $\boldsymbol{x}$ belongs to a cycle, then $\left\|\lfloor g\rfloor_{\omega}(\boldsymbol{x})\right\|_{\infty}=\|\boldsymbol{x}\|_{\infty}$.

Thanks to Lemma 4, we can also prove that the cycles of non expansive QLTs are 'not too far' from the origin. Thereby, we extend to the general case a result that was obtained in [4] for the $2 \mathrm{D}$ space.

Theorem 2. Consider a $Q L T\lfloor g\rfloor_{\omega}$ such that $\frac{1}{\omega} g_{0}$ is contracting and note $s=$ $\max \left(\frac{\|\boldsymbol{v}\|_{\infty}}{\omega}, 1-\frac{\|\boldsymbol{v}\|_{\infty}}{\omega}\right)$. If $\boldsymbol{x}$ belongs to a cycle then $\|\boldsymbol{x}\|_{\infty} \leq \frac{s}{1-\left\|g_{0}\right\|_{\infty} / \omega}$, the inequality being strict if $s>\frac{1}{2}$.

Proof. Let $g_{1}=(1 / \omega) g_{0}$. From lemma 4 , inductively we get

$$
\lfloor g\rfloor_{\omega}{ }^{k}(\boldsymbol{x})=g_{1}^{k}(\boldsymbol{x})+g_{1}^{k-1}\left(d_{1}(\boldsymbol{x})\right)+g_{1}^{k-2}\left(d_{2}(\boldsymbol{x})\right)+\cdots+g_{1}\left(d_{k-1}(\boldsymbol{x})\right)+d_{k}(\boldsymbol{x})
$$


with $\left\|d_{i}(\boldsymbol{x})\right\|_{\infty} \leq s$ for $i=1,2, \ldots, k$ each inequality being strict if $s>\frac{1}{2}$. It follows that

$$
\begin{aligned}
\left\|\lfloor\rfloor_{\omega}{ }^{k}(\boldsymbol{x})\right\|_{\infty} & \leq\left\|g_{1}^{k}(\boldsymbol{x})\right\|_{\infty}+\sum_{i=1}^{k}\left\|g_{1}^{k-i}\left(d_{i}(\boldsymbol{x})\right)\right\|_{\infty} \\
& \leq\left\|g_{1}\right\|_{\infty}^{k}\|\boldsymbol{x}\|_{\infty}+\sum_{i=1}^{k}\left\|g_{1}\right\|_{\infty}^{k-i}\left\|d_{i}(\boldsymbol{x})\right\|_{\infty} \\
& \leq\left\|g_{1}\right\|_{\infty}^{k}\|\boldsymbol{x}\|_{\infty}+s \sum_{i=1}^{k}\left\|g_{1}\right\|_{\infty}^{k-i} \\
& \leq\left\|g_{1}\right\|_{\infty}^{k}\|\boldsymbol{x}\|_{\infty}+s \frac{1-\left\|g_{1}\right\|_{\infty}^{k}}{1-\left\|g_{1}\right\|_{\infty}} .
\end{aligned}
$$

If $\boldsymbol{x}$ belongs to a cycle, it exits $k \in \mathbb{N}$ such that $\lfloor g\rfloor_{\omega}{ }^{k}(\boldsymbol{x})=\boldsymbol{x}$ and so

$$
\|\boldsymbol{x}\|_{\infty}\left(1-\left\|g_{1}\right\|_{\infty}^{k}\right) \leq s \frac{1-\left\|g_{1}\right\|_{\infty}^{k}}{1-\left\|g_{1}\right\|_{\infty}} .
$$

Finally, $\|\boldsymbol{x}\|_{\infty} \leq s /\left(1-\left\|g_{1}\right\|_{\infty}\right)$, the inequality being strict if $s>\frac{1}{2}$.

As noted in Remark 1, the points of a cycle of a non expansive QLT share the same infinite norm. Then, the following lemma shows that, for contracting QLTs with null constant part, those points cannot lie in the first hyperoctant.

Lemma 5. Let $\lfloor g\rfloor_{\omega}$ a $Q L T$ such that $\left\|\frac{1}{\omega} g_{0}\right\|_{\infty}<1$ and $\boldsymbol{v}=\mathbf{0}$. Consider $\boldsymbol{y}=$ $\lfloor g\rfloor_{\omega}(\boldsymbol{x})$ where $\boldsymbol{x} \in \mathbb{Z}^{n}$. If $\|\boldsymbol{y}\|_{\infty}=\|\boldsymbol{x}\|_{\infty}$, then the coordinates of $\boldsymbol{y}$ with maximal absolute value are negative.

Proof. From the hypotheses, we have

$$
\left\|\frac{1}{\omega} g_{0}(\boldsymbol{x})\right\|_{\infty} \leq\left\|\frac{1}{\omega} g_{0}\right\|_{\infty}\|\boldsymbol{x}\|_{\infty}<\|\boldsymbol{y}\|_{\infty} .
$$

Thus, any positive coordinate of $\frac{1}{\omega} g_{0}(\boldsymbol{x})$ is less than $\|\boldsymbol{y}\|_{\infty}$ which prove the result.

Corollary 3 applies Theorem 2 to two common particular cases: $\|\boldsymbol{v}\|_{\infty}=\omega / 2$ (rounding half up) and $\boldsymbol{v}=\mathbf{0}$ (rounding down). In this corollary and in the following theorem we denote by $S_{n-1}$ the unit sphere $\left\{\boldsymbol{x} \mid\|\boldsymbol{x}\|_{\infty}=1\right\}$.

Corollary 3. Let $\lfloor g\rfloor_{\omega}$ be a $Q L T$.

- If $\|\boldsymbol{v}\|_{\infty}=\frac{\omega}{2}$ and $\left\|g_{0}\right\|_{\infty}<\frac{3}{4} \omega$ then any cycle is included in the unit sphere $S_{n-1}$.

- If $\boldsymbol{v}=\mathbf{0}$ and $\left\|g_{0}\right\|_{\infty}<\frac{1}{2} \omega$, then any cycle is included in the unit cube $\{-1,0\}^{n}$.

Proof. The first assertion is a direct consequence of Theorem 2. The second assertion is a consequence of Lemma 5 and Theorem 2. 
Eventually with the next theorem we show that not only the cycles of a non expansive QLT are not too far from the origin but moreover one of these cycles (if such a cycle exists) lies in the unit sphere $S_{n-1}$. Thereby, we solve a conjecture that was stated in [4] for the 2D case.

Theorem 3. Any $Q L T\lfloor g\rfloor_{\omega}$ derived from a non expansive linear transformation $(1 / \omega) g_{0}$ has a cycle in the unit sphere $S_{n-1}$ or has no cycle.

In order to prove the theorem we need the following technical lemma.

Lemma 6. Let $\lfloor g\rfloor_{\omega}$ be a QLT such that $\left\|\frac{1}{\omega} g_{0}\right\|_{\infty} \leq 1$. Let $\boldsymbol{x} \in \mathbb{Z}^{n} \backslash\{\mathbf{0}\}, \boldsymbol{p} \in$ $S_{n-1}$ be such that $\|\boldsymbol{x}-\boldsymbol{p}\|_{\infty}$ is minimal. If $\boldsymbol{y}=\lfloor g\rfloor_{\omega}(\boldsymbol{x})$ has the same norm as $\boldsymbol{x}$, then $\boldsymbol{q}=\lfloor g\rfloor_{\omega}(\boldsymbol{p})$ is in $S_{n-1}$ and $\|\boldsymbol{y}-\boldsymbol{q}\|_{\infty}$ is minimal.

Proof. As $\boldsymbol{p} \in S_{n-1}$, Corollary 2 induces that $\boldsymbol{q} \in S_{n-1} \cup\{\mathbf{0}\}$. Moreover, again from Corollary 2, we derive that:

$$
\begin{aligned}
\|\boldsymbol{y}-\boldsymbol{q}\|_{\infty} & =\left\|\lfloor g(\boldsymbol{x})\rfloor_{\omega}-\lfloor g(\boldsymbol{p})\rfloor_{\omega}\right\|_{\infty} \\
& =\left\|\left\lfloor g_{0}(\boldsymbol{x}-\boldsymbol{p})+g(\boldsymbol{p})\right\rfloor_{\omega}-\lfloor g(\boldsymbol{p})\rfloor_{\omega}\right\|_{\infty} \\
& =\left\|\left\lfloor g_{0}(\boldsymbol{x}-\boldsymbol{p})+\boldsymbol{u}\right\rfloor_{\omega}\right\|_{\infty} \quad \text { where } \boldsymbol{u}=g(\boldsymbol{p})-\omega\lfloor g(\boldsymbol{p})\rfloor_{\omega} \\
& \leq\|\boldsymbol{x}-\boldsymbol{p}\|_{\infty} \quad \text { for } \boldsymbol{x} \mapsto g_{0}(\boldsymbol{x})+\boldsymbol{u} \text { is a non expansive QLT. }
\end{aligned}
$$

Since $\|\boldsymbol{x}-\boldsymbol{p}\|_{\infty}$ is minimal and $\boldsymbol{p} \in S_{n-1}$, one has $\|\boldsymbol{x}-\boldsymbol{p}\|_{\infty}=\|\boldsymbol{x}\|_{\infty}-1=$ $\|\boldsymbol{y}\|_{\infty}-1$. Then,

$$
\|\boldsymbol{y}-\boldsymbol{q}\|_{\infty}<\|\boldsymbol{y}\|_{\infty}
$$

We conclude that $\boldsymbol{q} \neq \mathbf{0}$ so that $\boldsymbol{q} \in S_{n-1}$ and $\|\boldsymbol{y}-\boldsymbol{q}\|_{\infty}$ is minimal.

Proof (Theorem 3). Consider $\boldsymbol{x}$ a point of a cycle and a point $\boldsymbol{p}$ defined as in lemma 6 and consider the sequences $\boldsymbol{x}_{\boldsymbol{i}}=\lfloor g\rfloor_{\omega}{ }^{i}(\boldsymbol{x})$ and $\boldsymbol{p}_{\boldsymbol{i}}=\lfloor g\rfloor_{\omega}{ }^{i}(\boldsymbol{p})$. For each $i \in \mathbb{N}$ the points $\boldsymbol{x}_{\boldsymbol{i}}$ and $\boldsymbol{p}_{\boldsymbol{i}}$ verify the condition of lemma 6 . The sequence $\boldsymbol{p}_{\boldsymbol{i}}$ is then an infinite sequence of points in $S_{n-1}$. But it exits a finite number of points in $S_{n-1}$ so there is a cycle among these points.

Remark 2. If $\boldsymbol{v}=\mathbf{0}$ and $\left\|g_{0}\right\|_{\infty}<\boldsymbol{\omega}$, from Lemma 5 and Theorem 3, we derive that there exists a cycle in the unit cube $\{-1,0\}^{n}$.

\section{Conclusion}

In this paper we completed and improved some theoretical results abouts QATs. We gave a closed formula for the period of the tiles generated by QATs and proved a conjecture on the localization of their cycles. We plan to use these results in a multigrid and multiprecision framework that we are developing in a joint work between Poitiers University and Strasbourg University [1]. The goal of this project is to build a formal theory in which the consequences of the change of universe between affine and quasi-affine transforms, that is between real numbers and integers, is precisely described so that one could choose a calculus precision and an image resolution to ensure a set of properties up to 
a tolerance threshold. For instance, at any precision and resolution, almost all quasi-affine rotations have non-singular tiles. Nevertheless, it would be desirable firstly to prove that though the size in pixels of the tiles grows with the precision, their 'true' sizes, taking into account the space resolution, tend toward zero and secondly to bound the convergence speed. Alike, we have to ensure for example that, despite the increasing complexity of cycles of quasi-affine rotations as the precision goes to infinity, they converge, together with fixed points, toward the center of the rotation when the pair precision - resolution tends toward infinity.

\section{References}

1. Andres, E., Da Col, M., Fuchs, L., Largeteau-Skapin, G., Magaud, N., Mazo, L., Zrour, R.: Les omega-aqa : Représentation discrète des applications affines. In: Passat, N. (ed.) GéoDis (2014)

2. Blot, V., Coeurjolly, D.: Quasi-affine transformation in higher dimension. In: Brlek, S., Reutenauer, C., Provenal, X. (eds.) DGCI, LNCS, vol. 5810, pp. 493-504. Springer (2009)

3. Coeurjolly, D., Blot, V., Jacob-Da Col, M.A.: Quasi-affine transformation in 3-d: Theory and algorithms. In: Wiederhold, P., Barneva, R. (eds.) IWCIA, LNCS, vol. 5852, pp. 68-81. Springer (2009)

4. JACOB, M.A.: Applications quasi-affines. Ph.D. thesis, Université Louis Pasteur, Strasbourg (1993)

5. Jacob-Da Col, M.A., Tellier, P.: Quasi-linear transformations and discrete tilings. Theoretical Computer Science 410(2123), 2126 - 2134 (2009)

6. JACOB-DA COL, M.A.: Applications quasi-affines et pavages du plan discret. In: Theoretical Computer Science. vol. 259, pp. 245-269. Elsiever Science (2001), available in english at https://dpt-info.u-strasbg.fr/ jacob

7. M-A. JАСОB, J.P.R.: Gaussian numeration systems. In: 5ème colloque DGCI. pp. 37-47 (1995), available at https://dpt-info.u-strasbg.fr/ jacob

8. Nehlig, P.: Applications Affines Discrètes et Antialiassage. Ph.D. thesis, Université Louis Pasteur, Strasbourg (1992)

9. Nehlig, P.: Applications quasi-affines : pavages par images réciproques. In: Theoretical Computer Science. vol. 156, pp. 1-38. Elsiever Science (1995)

10. Ngo, P., Passat, N., Kenmochi, Y., Talbot, H.: Topology-preserving rigid transformation of $2 \mathrm{~d}$ digital images. Image Processing, IEEE Transactions on 23(2), 885-897 (2014)

11. Nouvel, B., Rémila, E.: Configurations induced by discrete rotations: Periodicity and quasi-periodicity properties. Discrete Applied Mathematics 147(2), 325-343 (2005)

12. Nouvel, B., Rmila, E.: Characterization of bijective discretized rotations. In: Klette, R., uni, J. (eds.) Combinatorial Image Analysis, Lecture Notes in Computer Science, vol. 3322, pp. 248-259. Springer (2005) 